\title{
Author Correction: H3K64 trimethylation marks heterochromatin and is dynamically remodeled during developmental reprogramming
}

Sylvain Daujat, Thomas Weiss, Fabio Mohn, Ulrike C Lange, Céline Ziegler-Birling, Ulrike Zeissler, Michael Lappe,

Dirk Schübeler, Maria-Elena Torres-Padilla and Robert Schneider

Correction to: Nature Structural \& Molecular Biology https://doi.org/10.1038/nsmb.1629, published online 28 June 2009.

In this article, the Ponceau staining presented in Fig. $1 \mathrm{~b}$ (right, bottom) does not follow best practices for figure preparation since it inadvertently included duplications from the Ponceau staining presented in Supplementary Fig. 1b (for which the same preparation of nucleosomes from HeLa cells had been used). A new Fig. 1b is provided in the Author Correction with a different H3K64me3 immunoblot (top) and Ponceau staining (bottom); both the immunoblot and Ponceau membranes contain the same batch of HeLa nucleosomes, but it is a different batch than that in the original Fig. 1b. The interpretation of Fig. $1 \mathrm{~b}$ is not affected by this mistake. We apologize for any confusion this may have caused.

We describe here the experimental procedure for the experiment in Fig. 1b and Supplementary Fig. 1b,e in more detail. Equal amounts of HeLa nucleosomes were loaded on all lanes, separated by SDS-PAGE, transferred to nitrocellulose membranes and stained with Ponceau. Membranes were then cut into strips (black dotted lines in the new Fig. 1b), and each strip was individually incubated with H3K64me3 antibody preadsorbed with 100 pmoles/ml of the indicated H3 and $\mathrm{H} 4$ peptides. Ponceau staining of a membrane with the same batch and quantity of HeLa nucleosomes loaded is shown as reference (bottom). Note that, because the strips were randomized to avoid biases, the lanes in the Ponceau staining may not correspond directly to the ones shown in the immunoblot.
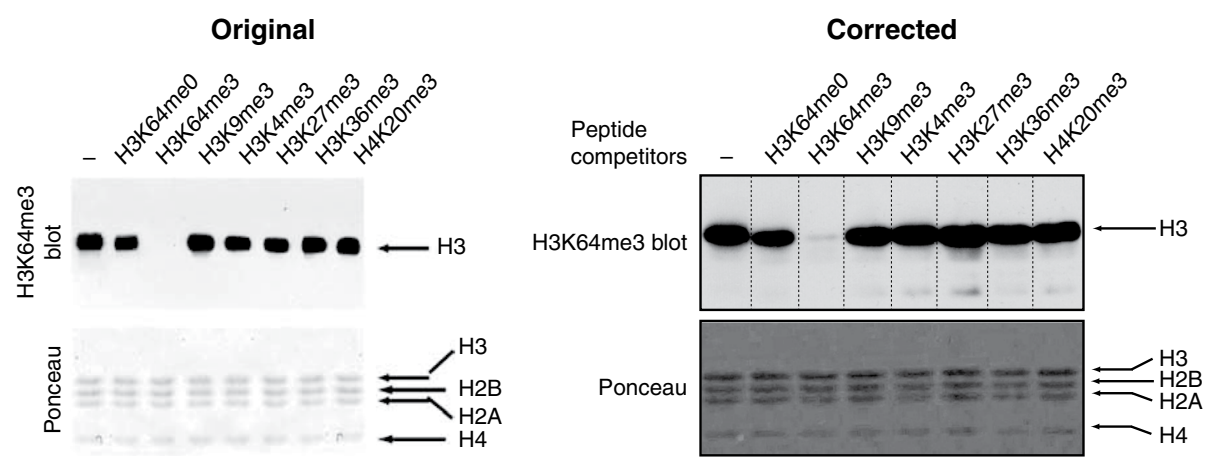

Fig. 1 | Original and Corrected.

Published online: 11 July 2018

https://doi.org/10.1038/s41594-018-0090-0

\section{Author Correction: Structure of the core of the type III secretion system export apparatus}

Lucas Kuhlen (D), Patrizia Abrusci, Steven Johnson (D), Joseph Gault (D), Justin Deme, Joseph Caesar, Tobias Dietsche (D), Mehari Tesfazgi Mebrhatu, Tariq Ganief, Boris Macek, Samuel Wagner (D), Carol V. Robinson and Susan M. Lea (DD

Correction to: Nature Structural \& Molecular Biology https://doi.org/10.1038/s41594-018-0086-9, published online 2 July 2018.

In the version of this article initially published, the PDB code associated with the study was given as 6F2E but should have been $6 \mathrm{~F} 2 \mathrm{D}$ in Table 1 and the data availability statement. The error has been corrected in the HTML and PDF versions of the article. 